\title{
Quelques remarques sur la crue exceptionnelle d'octobre 1960 dans l'ouest du Massif Central
}

\author{
Comments on the exceptional \\ October 1960 flood \\ in the western Central Massif
}

\author{
par le Professeur Maurice PARDE
}

\begin{abstract}
M. le Professeur M. Pardé, qui n'avait pu assister le 5 mai 1961 à la réunion de la Commission pour l'étude des débits de crues, avait cependant adressé d'intéressantes remarques sur les mémoires présentés.

M. Jean DE Beauregard, ingénieur au Service des programmes d'Electricité de France, a bien voulu résumer ces remarques dans le texte ci-après.
\end{abstract}

I. - Tout d'abord j'ai été fort intéressé de lire sous la plume de M. Fontaine que le mécanisme atmosphérique responsable de la grande pluie, a été fort insolite. M. Fontaine montre très bien, avec figures et autres données à l'appui, que la situation météorologique, l'emplacement des centres d'action et les espaces occupés par les diverses masses d'air dès le 2 octobre et encore plus le 3, au matin, semblaient devoir annoncer une crue méditerranéenne cévenole, selon notre terminologie. Et M. Fontarne montre encore que le 5 et le 6 octobre suivants, les éléments atmosphériques essentiels étaient à peu près les mêmes, tout au moins en apparence.

Cette fois, il s'est produit une averse méditerranéenne typique (selon mes qualifications) sur le bassin du Rhône. Je ne reviens pas sur les explications extrêmement intéressantes que présente M. Fontaine. Mais je veux, peut-être avec amour-propre, faire remarquer que le 2 octobre j'avais annoncé, presque à grands fracas, à qui voulait m'entendre, la possibilité et la probabilité d'une crue méditerranéenne extensive ou cévenole sur le bassin inférieur du Rhône, et je crois pouvoir dire que fort souvent mes pronostics de ce genre, fondés sur l'aspect du ciel, les températures, la direction des vents et les cartes barométriques, se révèlent point absurdes.

Cela montre d'une part que les situations atmosphériques considérées sont extrêmement typiques et bien reconnaissables pour des personnes attentives et, d'autre part, que « l'affaire peut rater» ou plutôt se passer ailleurs. Déjà j'avais noté l'extension d'une averse scmblable occupant une surface étroite et pointue vers le nord - nord-ouest jusque vers Limoges. La chose arriva lors de la pluie méditerranéenne mémorable de mars 1930. Mais, cette fois, le coup n'avait point avorté sur le Languedoc et l'extension pluvieuse vers le nord n'a été qu'un hors-d'œuvre, 
d'ailleurs très remarquable. Je constate que cet événement n'a point échappé à l'étude rétrospective de M. Fontarne.

II. - En ce qui concerne les crues ellesmêmes, il y a dans l'ensemble des mémoires présentés à la séance, une petite lacune. Ces textes n'indiquent point, ce me semble, quel fut le sort de la crue sur les cours principaux des grandes rivières en aval de la région la plus sinistrée. On a tout à fait raison d'utiliser le plus possible et en premier lieu les résultats obtenus aux stations de la $4^{\mathrm{e}}$ Circonscription Electrique et de l'E.D.F., stations pour lesquelles il existe des courbes de relations hauteurs-débits. Mais il semble indispensable de ne point oublier les vieux postes du Service ordinaire des Ponts et Chaussées, malgré l'absence ou l'insuffisance des données sûres en débits pour beaucoup d'entre eux. Car ces stations fonctionnent depuis un demi-siècle à un siècle pour la plupart. Les conditions d'écoulement semblent n'y avoir point trop changé dans l'ensemble et les variations hydrométriques et les niveaux maximaux en ces lieux permettent d'y comparer les crues récentes avec celles du passé. Ơn peut d'ailleurs émettre des approximations sur les débits eux-mêmes.

L'examen de ces niveaux nous montre avec précision comment la crue des 3-4 octobre s'est remarquablement atténuée sur les cours inférieurs des principales rivières; phénomène d'ailleurs évident à la lecture des journaux, malgré la médiocrité ou même la stupidité fréquente des nouvelles que ceux-ci présentent sur les crues. Il importe donc de donner sur ce point quelques informations numériques.

Le maximum de la Dordogne à Bergerac n'a point dépassé $8,70 \mathrm{~m}$ et, d'après les jaugeages de M. BARrière, on peut estimer le débit aux environs de $2500 \mathrm{~m}^{3}$, la Dordogne moyenne n'ayant débité en pointè que 1400 à $1500 \mathrm{~m}^{3}$ au plus à Domme au lieu de 3000 à 3500 sinon plus lors des crues records. A Bergerac, les flots de mars 1783 et de janvier 1728 ont pu représenter 4500 à $5000 \mathrm{~m}^{3}$. Le niv̌eau de 1728 aurait été de $12,48 \mathrm{~m}$. Sur la Vienne moyenne à Châtellerault, station principale, le maximum n'a pas atteint $4 \mathrm{~m}$ contre $6,77 \mathrm{~m}$ en février 1698 , environ $6,30 \mathrm{~m}$ à $6,40 \mathrm{~m}$ en juillet 1792 , mars 1913 , décembre 1944. La Vienne supérieure avait été d'ailleurs bien moins touchée que la Creuse. Pour le cours inférieur de celle-ci à la station principale de la Roche-Posay, le niveau d'octobre n'a point dépassé $5,60 \mathrm{~m}$ contre un record certain de $7,30 \mathrm{~m}$ en octobre 1896 , et peut-être $7,88 \mathrm{~m}$ d'après un repère, en juillet 1792 .

Approximativement, j'évalue le débit maximal en ces circonstances entre 1500 et $2000 \mathrm{~m}^{3}$. Je doute qu'il ait dépassé $1000 \mathrm{~m}^{3}$ en octobre
1960, alors qu'à Eguzon on avait eu $1150 \mathrm{~m}^{3}$. Puis à Nouatre, après le confluent des deux rivières, le maximum a fait $7,20 \mathrm{~m}$ contre $10,51 \mathrm{~m}$ (événement mémorable), en juillet 1792.

D'après un excellent jaugeage récent de M. BARriére, le maximum de 1960 a dû être de $1720 \mathrm{~m}^{3}$. En outre, la grandeur de l'extrapolation nécessaire gêne pour l'évaluation du maximum connu, celui de juillet 1792. Provisoirement, je le juge susceptible d'avoir représenté 3250 à $3500 \mathrm{~m}^{3}$.

Enfin si la Tardes a été relativement formidable, le Cher inférieur n'a eu qu'une crue banale, puisqu'elle n'a pas dépassé $3 \mathrm{~m}$ à Noyers contre 4,03 en mai 1940 et $4,36 \mathrm{~m}$ en mai-juin 1856 . Sans doute le débit n'a pas dû dépasser 500 à $550 \mathrm{~m}^{3}$ contre 1000 ou 1200 en mai 1856 (1600 selon les chiffres anciens du Service des Ponts et Chaussées).

III. - Autre remarque d'une grande importance au sujet des débits maximaux :

S'ils ont battu très gravement les records connus de mémoire d'homme sur la Corrèze, la Vézère, surtout en aval du confluent avec la Corrèze, puis sur la haute Creuse et la Tardes, ces débits en soi étaient assez peu imposants, tout d'abord par comparaison avec ceux des crues cévenoles. Nous rappelons que pour $1080 \mathrm{~km}^{2} \mathrm{le}$ Gardon à Ners le 30 septembre 1958 paraît bien avoir débité environ $5000 \mathrm{~m}^{3}$, à 10 ou $15 \%$ près, soit plus de 150 pour le coefficient $\mathrm{A}=\mathrm{Q} / \sqrt{\mathrm{S}}$, débit maximal divisé par la racine carrée de la surface réceptrice.

Or pour $950 \mathrm{~km}^{2}$ de la Corrèze à Brive, on a eu $750 \mathrm{~m}^{3}$, soit seulement $24,4 \mathrm{~A}$. Sur la Creuse à Eguzon, $1150 \mathrm{~m}^{3}$ pour $2400 \mathrm{~km}^{2}$ font 23,4 pour le coefficient. Les records de la Dordogne à Bergerac, puis du Lot à Cahors doivent donner 40 ou plus. Celui du Tarn à Montauban en mars 1930 a valu plus de 60. Si l'on prend de très petits bassins, la plus forte crue observée le 5 octobre, celle de la Vimbelle, à savoir $183 \mathrm{~m}^{3}$ pour $140 \mathrm{~km}^{2}$, semble bien piètre à côté des cataclysmes cévenols. Pour $140 \mathrm{~km}^{2}$, ceux-ci doivent pouvoir donner $1500 \mathrm{~m}^{3}$; puis, même dans des régions océaniques comme le bassin supérieur de la Moselle, ou celui de l'Ain, les débits maximaux semblent atteindre des chiffres supérieurs à ceux de la Corrèze, de la Creuse, etc.

A Epinal, pour $1200 \mathrm{~km}^{2}$ en gros, la crue de décembre 1947 a peut-être fait $1000 \mathrm{l} / \mathrm{s}$ par $\mathrm{km}^{2}$. Et. pour l'Ain à Cize-Bolozon, nous connaissons au moins trois crues (décembre 1918, février 1920, février 1957) qui ont dû débiter 750 à $800 \mathrm{l} / \mathrm{s}$ par $\mathrm{km}^{2}$ pour un peu plus de $2500 \mathrm{~km}^{2}$. Et cependant les pluies responsables dans le 
jour d'intensité maximale ont été moindres pour ces bassins que pour le plateau de Millevaches les 3-4 octobre 1960 .

IV. - Enfin, nous jugeons utiles quelques remarques sur les rapports entre les débits de la crue et les volumes totaux responsables. M. BARrière a calculé, pour quelques cours d'eau, dans les bassins de la Vézère et de la Corrèze surtout, les rapports entre les volumes liquides totaux les plus forts de trois jours et la pluie totale responsable. Et pour ma part, j'avais refait les mêmes calculs avec des méthodes très légèrement différentes.

Nos chiffres concordent pour presque toutes les stations, sauf pour celle de Bugeat où M. BARRIÈrE donne $56 \%$ et moi-même seulement $51 \%$. Je n'ai aucune raison de croire que mon chiffre est le meilleur. Cependant je croirais volontiers que, sur la haute Vézère, le rapport a pu être un peu plus faible que sur la Corrèze et la Vimbelle, en raison du gros pouvoir de rétention que doit posséder le bassin.

Sur la Corrèze donc, les rapports sont compris entre 0,52 et 0,55 et l'on a 0,50 sur la Tardes à Evaux, 0,67 sur la Luzège au pont des Bouyges et 0,63 sur le Cher à Teillet. Ces deux chiffres me surprennent quelque peu par leur grandeur, mais peut-être à tort.

De toutes façons, il me semble nécessaire de comparer les quotients d'écoulement de 3 jours à ceux de 8 à 10 jours. J'ai compté les volumes liquides pour ces durées, approximativement, au-dessus des débits sans cesse décroissants qui se seraient écoulés si la crue n'avait point eu lieu. Et j'ai extrapolé tant bien que mal les diagrammes des décroissances en ne tenant pas compte d'une petite recrudescence survenue quelques jours après la grande apophyse.

J'ai trouvé pour la Corrèze et la Vézère en gros 65 à $74 \%$. Ces chiffres sont élevés pour un début d'octobre, moment où d'habitude beaucoup d'eau s'infiltre en profondeur et où des valeurs inférieures à 60 et même $50 \%$ pour 8 à 10 jours sont courantes.

La grandeur des quotients d'écoulement obtenus a évidemment pour cause l'intensité exceptionnelle de l'averse, puis la saturation préalable par de fortes pluies en août et en septembre. D'ailleurs, cette imbition préparatoire du sol aurait pu être encore plus considérable. Quant au déficit d'écoulement de crue, il semble avoir atteint en 8 ou 10 jours en général 50 à $60 \mathrm{~mm}$, plus cependant pour la Diège au pont de Loubeix. Pour cette rivière, M. d'Harcourt trouve en 4 jours un coefficient d'écoulement de $36 \%$, et moi, pour 7 à 8 jours, j’obtiens à peu près $42 \%$. Le déficit serait d'une centaine de millimètres.
On doit donc supposer qu'en ce bassin la capacité de rétention durable est très grande. Pour celui de la Vézère à l'amont de Bugeat, cette rétention est cependant faible, puisque le quotient d'écoulement de 8 jours serait le plus fort de ceux que j'ai calculés, avec $74 \%$. Mais on doit avoir une opinion plus approfondie sur les modalités de la rétention, soit d'après l'examen des hydrogrammes unitaires puis combinés, soit en considérant comme j'ai l'habitude de le faire «les rapport de crue ». Ce sont les quotients des débits maximaux fluviaux spécifiques par les débits pluviaux moyens d'une certaine durée; les premiers chiffres tendent à rejoindre les seconds. Et si l'infiltration comme l'évaporation finissent par devenir faibles, le rapport de crue peut atteindre 90 à $100 \%$ dans de petits bassins très imperméables ou très saturés, surtout lors des très grandes crues de plein hiver.

Nous connaissons dans le monde des rapports de l'ordre de 80 à $90 \%$ ou $95 \%$, même pour des surfaces réceptrices de plusieurs milliers de $\mathrm{km}^{2}$. Or, pour la crue en question, les rapports ont été beaucoup moins remarquables. Nous avons trouvé par comparaison avec les débits pluviaux moyens de 24 heures, environ 70 ou $75 \%$ pour la Vimbelle, chiffre le plus gros de tous; puis 62 à $66 \%$ pour la Corrèze à Tulle; 54 à $57 \%$ pour la Corrèze à Corrèze; 51 à $54 \%$ à Brive, et seulement 43 à $45 \%$ pour la Diège, environ $40 \%$ pour la Vézère à Bugeat.

La plupart de ces chiffres sont relativement faibles si l'on songe à l'intensité remarquable de la pluie et au fait que 24 heures représentent une durée de deux à quatre fois égale sans doute au temps de concentration pour les stations examinées.

Si les coefficients d'écoulement en trois jours sont «honorables » et en huit jours relativement très forts, et puisque les rapports de crue sont modérés ou médiocres, une conclusion s'impose : les bassins supérieurs en question possèdent un pouvoir de rétention considérable à l'encontre des débits maximaux de pointe et même des ruissellements de un jour ou un peu plus. Mais une grande partie de l'eau retenue semble être restituée après 8 à 10 jours.

Donc cette rétention est peu profonde. Nous pensons qu'elle s'effectue sur une couche superficielle de la désagrégation, peu épaisse au-dessus des roches cristallines, en soi imperméables, du plateau de Millevaches. De nombreux replats ou bassins de pentes très faibles avec tourbières, fougères, herbes, etc., puis un reboisement considérable effectué sur le plateau de Millevaches, grâce à l'apostolat de mon vieil ami le forestier Marius Vazeilles, contribuent certainement à la 
forte valeur du débit infiltré ou hypodermique ou de ruissellement très ralenti.

D'après l'examen des rapports de crue, un fait décisif me semble indubitable; les capacités de rétention du plateau de Millevaches et même de sa périphérie, ont réduit de moitié peut-être les maximums de la Vézère supérieure; puis d'au moins 25 ou $30 \%$ ceux de la haute Corrèze, sensiblement moins ceux de la Vimbelle. Pour la Diège, l'atténuation a été certainement supérieure à 25 ou $30 \%$.

\section{CONCLUSION}

En somme, quoique désastreuses, les crues d'octobre 1960 onl été assez sensiblement inférieures à celles qui, sur d'autres terrains ou après une saturation plus forte encore, eussent résulté de pluies aussi remarquables.

D’une façon générale, si les excédents pluviaux observés en août, avaient eu lieu en septembre, le sol eut été beaucoup plus saturé et les maximums des crues eussent peut-être été supérieurs de 10 à 20 ou $25 \%$. Il va de soi que mes remarques sont basées sur des études insuffisamment approfondies; mais elles peuvent contenir une forte part de vérité.

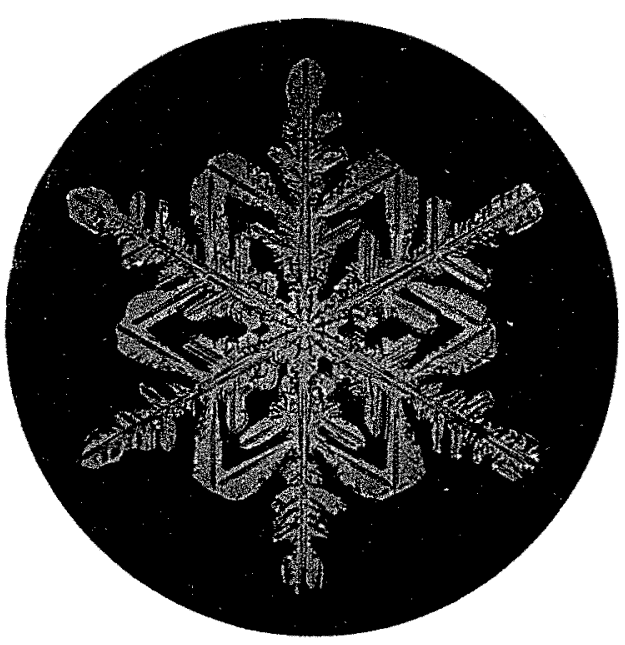

